\title{
Computerized tomography of the Thorax for surgical patients during the COVID-19 pandemic: Was it useful?
}

\author{
Ahmed Ismail ${ }^{1}$, Priya Sarkar², Balasundaram Muthiah², and Nuha Yassin² \\ ${ }^{1}$ Royal Wolverhampton Hospitals NHS Trust \\ ${ }^{2}$ New Cross Hospital
}

April 30, 2021

\begin{abstract}
Objectives Diagnostic challenges during the COVID-19 pandemic forced the radiology regulating body to adopt the use of CT Chest as a triage and diagnostic tool, which was subsequently abandoned. The Royal Wolverhampton hospital followed both protocols. Here, we investigate the evidence behind this decision within the context of surgical admissions during the COVID-19 peak in our hospital. Methods Retrospective data collection and analysis of all surgical admissions between the 1st of March to the 31st of May. Data was collected from the radiology and electronic portal looking into patients undergoing CT chest to diagnose the presence of C-19 as well as swab results. Results 78 patients fulfilled our inclusion criteria. The scan either confirmed the presence or absence (4, 63 patients) of C-19 but was sometimes inconclusive (11 patients). Comparing these to the results of the swabs; CT showed sensitivity $42.86 \%$, Specificity $97.92 \%$, and accuracy $90.91 \%$. In the inconclusive CT report group, chances of having a positive swab result were $45 \%$ : None of the scan results changed any of the surgical planning. Lymphocyte count in the context of surgical presentation did not have any statistical significance to predict the presence of C-19 $(\mathrm{P}=0.7)$. Cost implications on our cohort of patients for adding the chest $\mathrm{CT}$ is estimated to be around £31,000. Conclusion CT Thorax during the pandemic was a good negative predictor but had limited diagnostic value and did not change patient management. Newer, faster techniques of PCR swabs and antibody testing would be a better and cheaper alternative. Advances in knowledge This paper provides evidence to support the decision from the regulatory bodies not to use CT scan as a screening tool for COVID 19 diagnosis.
\end{abstract}

\section{Hosted file}

CT Thorax pub ver 1.pdf available at https://authorea.com/users/411073/articles/520224computerized-tomography-of-the-thorax-for-surgical-patients-during-the-covid-19pandemic-was-it-useful

\section{Hosted file}

Tables and Graphes .pdf available at https://authorea.com/users/411073/articles/520224computerized-tomography-of-the-thorax-for-surgical-patients-during-the-covid-19pandemic-was-it-useful 